\title{
Adiabatic quantum state manipulation of single trapped atoms
}

\author{
M. Khudaverdyan, W. Alt, I. Dotsenko, L. Förster, S. Kuhr, D. Meschede, Y. Miroshnychenko, \\ D. Schrader, and A. Rauschenbeutel* \\ Institut für Angewandte Physik, Universität Bonn, Wegelerstraße 8, D-53115 Bonn, Germany
}

(Received 15 November 2004; published 23 March 2005)

\begin{abstract}
We use microwave-induced adiabatic passages for selective spin flips within a string of optically trapped individual neutral Cs atoms. We position-dependently shift the atomic transition frequency with a magnetic field gradient. To flip the spin of a selected atom, we optically measure its position and sweep the microwave frequency across its respective resonance frequency. We analyze the addressing resolution and the experimental robustness of this scheme. Furthermore, we show that adiabatic spin flips can also be induced with a fixed microwave frequency by deterministically transporting the atoms across the position of resonance.
\end{abstract}

DOI: 10.1103/PhysRevA.71.031404

PACS number(s): 42.50.-p, 03.67.Lx, 39.25.+k, 32.80.Qk

Adiabatic passages (APs) [1] are an interesting alternative to purely resonant interaction for controlling the quantum state of atoms. They rely on the fact that the coupled atomfield system remains in its instantaneous eigenstate if the variation of its parameters (atom-field detuning and field strength) is sufficiently slow and smooth. One can thus adiabatically transfer a system from an initial to a final state and, under certain conditions, fluctuations of the parameters will not affect the outcome of the AP. The pioneering works concerning APs were performed in nuclear magnetism to achieve inversion of a spin system [2]. The first application of this method in the optical domain was realized in [3] to invert the population in $\mathrm{NH}_{3}$ molecules. Ever since, a multitude of different AP techniques have been proposed and successfully realized [4]. It has also been shown that APs can be used to robustly prepare superpositions of energy eigenstates [5]. Furthermore, it was proposed to prepare entanglement and implement quantum logic operations through adiabatic processes $[6,7]$.

We have recently demonstrated that a string of neutral Cs atoms stored in a standing-wave optical dipole trap can be used to realize a quantum register [8]. There, quantum information was written into the two Cs hyperfine ground states by subjecting selected atoms to resonant microwave pulses. Here, we report on the realization of an adiabatic method for flipping the states of individual atoms out of a string. As in [8], the atoms are discriminated through a positiondependent transition frequency. The APs are accomplished by sweeping the microwave frequency across the resonance frequency of the respective atom. We investigate the performance of our method by recording AP spectra of a few, as well as of single, atoms, yielding high-quality data in perfect agreement with theory. The spatial discrimination of this scheme is comparable to resonant addressing. At the same time, the method is much more robust than in the resonant case. It is therefore a useful tool for the manipulation and control of our quantum register.

Combining fixed-frequency microwave pulses with our "optical conveyor belt" technique $[9,10]$ inside a magnetic-

\footnotetext{
*Electronic address: rauschenbeutel@iap.uni-bonn.de
}

field gradient, we furthermore realize APs by transporting atoms across the position of resonance. In this experiment, the atom-field coupling and the position dependence of the transition frequency are chosen such that the dynamics of the system is similar to that of atoms coupled to the mode of an optical high-finesse Fabry-Perot resonator [11]. We show that our transport procedure is sufficiently smooth to guarantee adiabaticity over a large range of parameters, indicating that motion-induced APs could effectively be used in our system to control the atom-field interaction in cavity QED experiments.

We use a specially designed magneto-optical trap (MOT) as a source of a well-defined number of cold cesium atoms. Fluorescence light from the MOT is collected by an objective lens and is imaged onto the photocathode of an intensified charge-coupled device camera (ICCD) and onto an avalanche photodiode (APD), see Fig. 1. Whereas the APD signal allows us to monitor the number of atoms, the ICCD provides us with information about their positions [12]. The atoms are transferred from the MOT to a standing-wave dipole trap $[9,10]$, generated by two counterpropagating far red-detuned laser beams with a wavelength of $\lambda=1064 \mathrm{~nm}$. They interfere and produce a chain of potential wells of typically $1 \mathrm{mK}$ depth. Further details can be found in previous publications $[10,13]$.

As qubit states we employ the outermost Zeeman sublev-

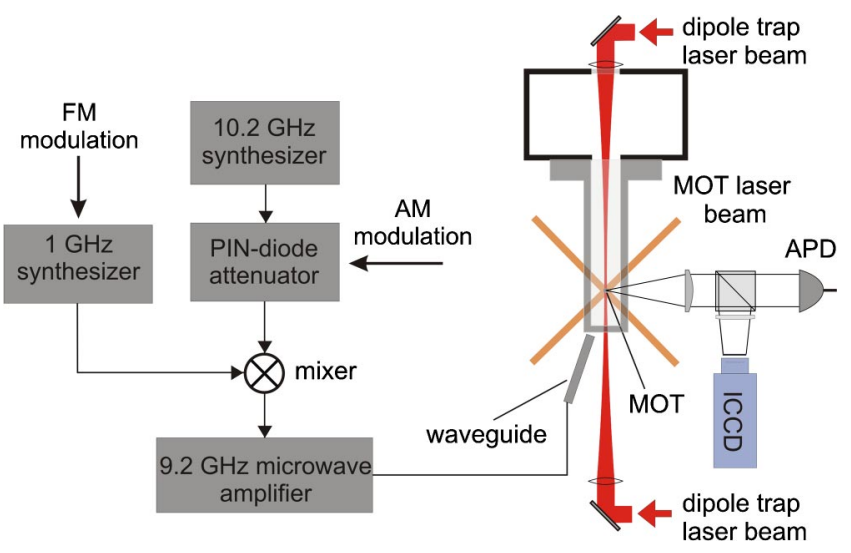

FIG. 1. Experimental setup. See text for details. 
els $\left|F=4, m_{F}=4\right\rangle(|0\rangle)$ and $\left|F=3, m_{F}=3\right\rangle(|1\rangle)$ of the two $6 S_{1 / 2}$ hyperfine ground states with a transition frequency $\omega_{\text {at }} / 2 \pi$ near $9.2 \mathrm{GHz}$. In order to spectroscopically distinguish atoms at different positions along the dipole trap axis, we apply a magnetic-field gradient of $13 \mathrm{G} / \mathrm{cm}$ along this axis. This results in a position-dependent transition frequency between states $|0\rangle$ and $|1\rangle$ of $\partial_{x} \omega_{\mathrm{at}} / 2 \pi$ $=3.2 \mathrm{kHz} / \mu \mathrm{m}[8]$.

Each experimental sequence starts by loading atoms into the MOT and transferring them to the dipole trap. In order to further lower the temperature of the atoms in the trapping potential an optical molasses is switched on shortly after the transfer. Then the trap depth is lowered from $1 \mathrm{mK}$ to $140 \mu \mathrm{K}$ in order to reduce the differential light shift (see below). A guiding magnetic field of $4 \mathrm{G}$ is applied along the dipole trap axis, which shifts $\omega_{\text {at }} / 2 \pi$ by $9.8 \mathrm{MHz}$. Optical pumping to the state $|0\rangle$ is accomplished by exposing the atoms to a $\sigma^{+}$-polarized laser beam along the guiding field, resonant with the $F=4 \leftrightarrow F^{\prime}=4$ transition, together with a repumping laser beam on the $F=3 \leftrightarrow F^{\prime}=4$ transition.

The microwave radiation for the APs is generated using two frequency synthesizers (see Fig. 1). The first one (Agilent $8375 \mathrm{~A}$ ) generates a signal at $10.2 \mathrm{GHz}$. Its output is sent through a $p$ - $i$ - $n$-diode attenuator that allows a fast modulation of the signal amplitude (AM). The second generator (Rohde \& Schwarz SML02) is frequency modulated (FM) and operates at $1 \mathrm{GHz}$. Both signals are mixed and the difference frequency at $9.2 \mathrm{GHz}$ is amplified to $36 \mathrm{dBm}$. Finally, it is sent through an open-ended waveguide and directed to the trapped atoms. This microwave setup combines the excellent frequency stability of the $10.2-\mathrm{GHz}$ source with the phase-continuous frequency sweeping capacity of the 1 $-\mathrm{GHz}$ source.

The computer-generated FM and AM control signals allow us to realize microwave pulses of arbitrary shapes. For inducing APs, we have chosen the pulse shape suggested in [14]

$$
\begin{gathered}
\Omega_{\mathrm{R}}(t)=\Omega_{\max } \sin ^{2}\left(\pi t / t_{\mathrm{p}}\right), \\
\delta(t)=\delta_{\mathrm{c}}+\operatorname{sgn}\left(t-t_{\mathrm{p}} / 2\right) \delta_{\max } \sqrt{1-\sin ^{4}\left(\pi t / t_{\mathrm{p}}\right)}
\end{gathered}
$$

for $0 \leqslant t \leqslant t_{\mathrm{p}}$. Here, $\Omega_{\max }$ is the maximum value for the Rabi frequency, $\delta(t)=\omega(t)-\omega_{\text {at }}$ is the detuning of the microwave frequency from atomic resonance, $\pm \delta_{\max }$ is the span of the frequency sweep around the central detuning $\delta_{\mathrm{c}}$, and $t_{\mathrm{p}}$ is the pulse duration.

The population transfer efficiency of the APs depends on the adiabaticity of the frequency sweep, which is reflected by the condition [15]

$$
\frac{\left|\dot{\delta}(t) \Omega_{\mathrm{R}}(t)-\delta(t) \dot{\Omega}_{\mathrm{R}}(t)\right|}{2\left(\Omega_{\mathrm{R}}(t)^{2}+\delta(t)^{2}\right)^{3 / 2}} \ll 1 .
$$

The pulse shape of Eq. (1) fulfills this adiabaticity condition over a wide range of the central detuning $\delta_{\mathrm{c}}$.

We first demonstrate adiabatic population transfer in a homogeneous magnetic field. Atoms are loaded into the dipole trap and are initialized in state $|0\rangle$. Then the frequencymodulated microwave pulse is applied. The final state of the

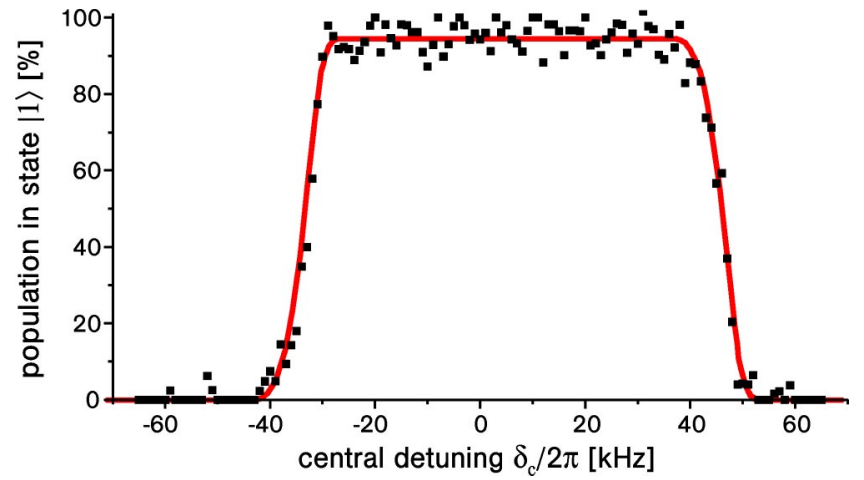

FIG. 2. Spectrum of adiabatic population transfer. Each data point shows the average population transfer to state $|1\rangle$ for ten shots with about five atoms each. The solid line is a theoretical fit, derived from optical Bloch equations. The maximum population transfer efficiency predicted by theory is $100 \%$. The fit yields $95 \pm 0.4 \%$, limited mainly by imperfect initialization of state $|0\rangle$, which depends critically on the polarization purity of the optical pumping laser.

atoms is detected using a "push-out" laser beam [16]. It expels atoms in $|0\rangle$ out of the trap with $99 \%$ efficiency, while atoms in $|1\rangle$ remain trapped with a probability higher than $99 \%$. The final number of atoms is revealed by transferring them back into the MOT and by detecting their fluorescence. The recorded spectrum is presented in Fig. 2, using the following microwave-pulse parameters: $\Omega_{\max } / 2 \pi=28 \mathrm{kHz}$, $\delta_{\max } / 2 \pi=40 \mathrm{kHz}$, and $t_{\mathrm{p}}=2 \mathrm{~ms}$. We have stepped the central detuning $\delta_{\mathrm{c}} / 2 \pi$ from -65 to $65 \mathrm{kHz}$ in steps of $1 \mathrm{kHz}$.

The wide plateau in Fig. 2 shows a population transfer efficiency $P_{1}>90 \%$ for $-30 \mathrm{kHz}<\delta_{\mathrm{c}} / 2 \pi<40 \mathrm{kHz}$. It is constant over a large interval of the central detuning demonstrating robustness of the spin-flip efficiency with respect to frequency drifts and fluctuations. Beyond this frequency interval the efficiency rapidly drops to zero. The asymmetry of the spectrum is due to an inhomogeneous broadening of the atomic resonance frequency, which is caused by the energydependent differential light shift $\hbar \delta_{\mathrm{ls}}=\Delta E_{0}-\Delta E_{1}$ of the individual trapped atoms [16], where $\Delta E_{i}$ is the light shift of level $i=0,1$, caused by the dipole trap laser.

We model the experimental data by calculating the transfer efficiency $P_{1}\left(\delta_{\mathrm{c}}\right)$ using Bloch equations for the set of parameters used in the experiment. The thermal distribution of atoms is then accounted for by convoluting $P_{1}\left(\delta_{\mathrm{c}}\right)$ with the three-dimensional Boltzmann distribution of differential light shifts [16],

$$
p_{B}\left(\delta_{\mathrm{ls}}\right)=\frac{\left(\delta_{\mathrm{ls}}-\delta_{\mathrm{ls}}^{\max }\right)^{2}}{2 \delta_{\mathrm{th}}^{3}} \exp \left(-\frac{\delta_{\mathrm{ls}}-\delta_{\mathrm{ls}}^{\max }}{\delta_{\mathrm{th}}}\right),
$$

where $\delta_{\mathrm{ls}}^{\max }$ is the maximum differential light shift and $\delta_{\text {th }}$ is the change of the differential light shift when the energy of the atom in the trapping potential is increased by $k_{B} T$. The resulting function

$$
\widetilde{P}_{1}\left(\delta_{c}\right)=P_{\max } \int_{\delta_{\mathrm{ls}}^{\max }}^{0} p_{B}\left(\delta_{\mathrm{ls}}\right) P_{1}\left(\delta_{c}+\delta_{\mathrm{ls}}\right) d \delta_{\mathrm{ls}}
$$

is then fitted to the data, with the maximum population $P_{\max }=95 \%, \delta_{\mathrm{th}} / 2 \pi=1.7 \mathrm{kHz}$, and $\delta_{\mathrm{ls}}^{\max } / 2 \pi=-11 \mathrm{kHz}$ as fit 


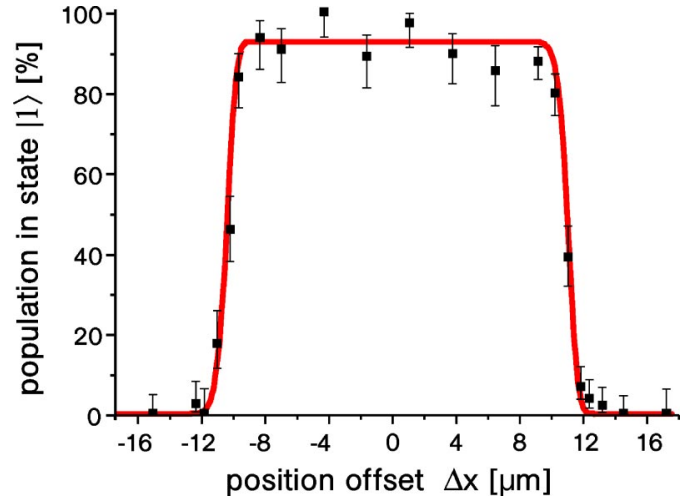

FIG. 3. Position-dependent adiabatic population transfer of individual atoms in an inhomogeneous magnetic field. The graph shows the population transfer induced by the AP as a function of the position offset $\Delta x$ along the trap axis. Each data point is obtained from about 40 single atom measurements. The solid line is a theoretical fit of Eq. (4) with $P_{\max }=93 \pm 1.7 \%$ limited by imperfect state initialization.

parameters. The fit (solid line in Fig. 2) is in excellent agreement with the experimental data.

In order to demonstrate position selective spin flips we now switch on the magnetic-field gradient. As in [8] we load one atom on average into the dipole trap, determine its position from a fluorescence image, and calculate the corresponding resonance frequency $\omega_{\mathrm{at}}$. After initializing the atom in state $|0\rangle$ we apply a microwave pulse of shape (1). We deliberately detune the central microwave frequency from $\omega_{\mathrm{at}}$ by $\delta_{\mathrm{c}}$. This corresponds to a position offset $\Delta x$ $=\delta_{\mathrm{c}} / \partial_{x} \omega_{\mathrm{at}}$. We detect the hyperfine state after application of the microwave pulse and record the single-atom population transfer as a function of the position offset $\Delta x$ along the trap axis.

The result of this measurement is presented in Fig. 3. Every data point is obtained from about 40 post-selected single atom measurements. As expected, the spectrum has a broad plateau with a population transfer efficiency above $90 \%$. At the edges this efficiency drops to zero within $3 \mu \mathrm{m}$. This addressing resolution is comparable to our resonant addressing scheme presented in [8]. However, the regime of high-efficiency population transfer extends over an interval that can be tuned by varying the span of the frequency sweep, $2 \delta_{\max }$, and that can thus be much larger $(18 \mu \mathrm{m}$ in Fig. 3) than in the resonant case, providing robustness against frequency drifts and fluctuations. In principle, the spatial resolution could further be increased by using a longer pulse duration $t_{\mathrm{p}}$. However, due to dephasing mechanisms [16], this would at some point decrease the maximum transfer efficiency.

We now examine the possibility of inducing adiabatic spin flips with a fixed microwave frequency by transporting the atoms across the position of resonance with our optical conveyor belt technique $[9,10]$. This option is particularly interesting for quantum information processing schemes in neutral atom cavity QED, where the atomic qubits interact through photon exchange inside an ultrahigh $Q$ optical resonator, see, e.g., [17]. In order to scale up such schemes to a larger number of atomic qubits, they will have to be shuttled into and out of the interaction zone. If this transport is sufficiently smooth to guarantee an adiabatic variation of the relevant parameters, one could therefore envision to adiabatically modulate the coupling strength of the atoms to the resonator by moving them into and out of the mode. This technique could even be used to reliably and robustly create entangled states through APs $[6,7]$.

Here, we show that our transport realizes this adiabaticity condition over a wide range of parameters. We move atoms in state $|0\rangle$ along the dipole trap in the magnetic-field gradient. Before transporting the atoms, the microwave radiation is adiabatically switched on. Then, during transportation, its amplitude is kept constant, corresponding to a Rabi frequency of $\Omega_{\mathrm{R}} / 2 \pi=26 \mathrm{kHz}$. The initial atom positions are distributed over $10 \mu \mathrm{m}$, due to the size of the MOT [12]. Because of the magnetic-field gradient this position spread corresponds to a frequency spread of $32 \mathrm{kHz}$. We therefore tune the microwave frequency $72 \mathrm{kHz}$ to the red side of the $|0\rangle \leftrightarrow|1\rangle$ transition, such that all atoms are initially out of resonance. We then transport the atoms over a distance of $d=132 \mu \mathrm{m}$ and thereby tune the atomic transition frequency by $\partial_{x} \omega_{\mathrm{at}} d / 2 \pi=420 \mathrm{kHz}$ to the red. These values are much larger than the initial detuning of the atoms and the effective width $L=2 \Omega_{\mathrm{R}} / \partial_{x} \omega_{\mathrm{at}}=16 \mu \mathrm{m}$ of the region of interaction. Therefore, after transport, all atoms, independently of their initial positions, have crossed the region of resonance and are again far detuned from the microwave frequency.

The atoms are transported using a uniform acceleration $a$, which undergoes a sign change at $d / 2$. Neglecting the thermal motion of the atoms inside the dipole trap, the resulting temporal variation of the detuning is then [see Fig. 4(a)]

$$
\delta(t)= \begin{cases}\delta_{r}+a \partial_{x} \omega_{\mathrm{at}} t^{2} / 2 & \text { for } t \leqslant \tau / 2 \\ \delta_{r}+a \partial_{x} \omega_{\mathrm{at}}\left(\frac{\tau^{2}}{4}-\frac{(t-\tau)^{2}}{2}\right) & \text { for } t>\tau / 2\end{cases}
$$

Here, $\delta_{\mathrm{r}}$ is a random initial detuning due to the spread of the atomic initial position, $a=4 d / \tau^{2}$ is the acceleration, $\partial_{x} \omega_{\text {at }}$ is the gradient of the atomic resonance frequency, and $\tau$ is the duration of transportation.

In Fig. 4(b) the percentage of atoms transferred to state $|1\rangle$ after transport is shown as a function of $1 / \tau \propto v$, where $v$ is the average speed with which the atoms cross the position of resonance. The population transfer has been normalized to the transportation efficiency, which has been measured independently, and exceeds $85 \%$ for $\tau$ ranging from $300 \mu$ s to $20 \mathrm{~ms}$. The maximum transfer efficiency is most probably limited by imperfect state initialization in this case. The solid line results from a numerical simulation and is normalized to $85 \%$. It predicts a reduced transfer efficiency for $1 / \tau$ $>3 \mathrm{~ms}^{-1}$ due to the loss of adiabaticity, in good agreement with the experiment. For larger $1 / \tau$ values, the experimental data falls off faster than predicted by theory. This is most probably due to the increasing excitation of axial oscillations of the atoms inside the potential wells caused by the abrupt changes in acceleration, which further reduce adiabaticity [10]. For $1 / \tau<0.05 \mathrm{~ms}^{-1}$ the experimental data also falls 
(a)

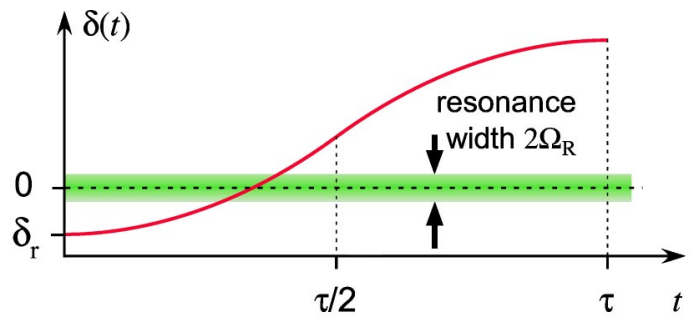

(b)

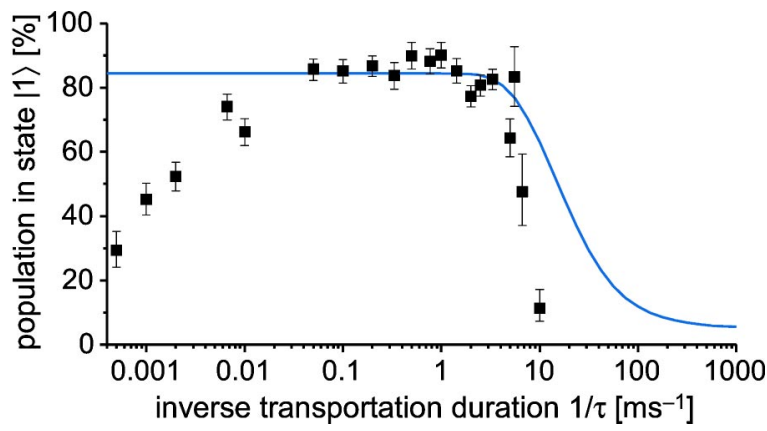

FIG. 4. Transport-induced adiabatic passages. (a) A sketch of the temporal variation of the atom-field detuning during the transport of duration $\tau$ [see Eq. (5)]. (b) The population in state $|1\rangle$ after transport as a function of $1 / \tau$. Each data point is an average over ten shots with about five atoms each. The solid line is the theoretical population transfer, neglecting the thermal motion of the atoms and decoherence of Rabi oscillations.

below the theoretical curve. This can be explained by the decay of the driven atomic dipole caused by various dephasing mechanisms [16].

Note that the effective width of the region of interaction $L$ is comparable to the diameter of the Gaussian mode of typical ultrahigh $Q$ Fabry-Perot resonators for cavity QED ex- periments [11]. Furthermore, the most promising schemes for the generation of entanglement in optical cavity QED rely on four-photon Raman processes. Based on the proposal in [17], we have calculated an effective Rabi frequency of $18 \mathrm{kHz}$ for this Raman process using optimized experimental parameters. This value is comparable with our microwave Rabi frequency $\Omega_{R}$. Our experimental results therefore indicate that our optical conveyor belt allows us to transport two atoms sufficiently smoothly through a resonator mode to realize entangling schemes based on cavity-assisted APs.

Summarizing, we have demonstrated that microwaveinduced adiabatic passages in a magnetic-field gradient provide an efficient and robust tool to manipulate the states of individual atoms in our neutral atom quantum register. The spatial resolution of this method is comparable with what can be achieved with resonant pulses under similar experimental conditions [8], while offering reduced sensitivity to fluctuations of experimental parameters. We have furthermore shown that adiabatic population transfer can also be realized with a fixed microwave frequency and amplitude by transporting the atoms across the resonance position using our optical conveyor belt technique. The wide range of transportation times for which efficient transfer occurs demonstrates that this scheme could indeed be used to adiabatically modulate the coupling of the atoms to spatially varying external fields. In particular in the context of cavity quantum electrodynamics, adiabatic entangling schemes, induced by the deterministic transport of two atoms through the resonator mode, appear to be within the scope of our experiment.

This work was supported by the Deutsche Forschungsgemeinschaft (SPP 1078) and the EC (IST/FET/QIPC project "QGATES"). I. D. acknowledges funding from INTAS. D. S. acknowledges funding by the Deutsche Telekom Stiftung.
[1] L. Allen and J. H. Eberly, Optical Resonance and Two-level Atoms (Wiley, New York, 1975).

[2] A. Abragam, The Principle of Nuclear Magnetism (Clarendon, Oxford, 1961).

[3] M. Loy, Phys. Rev. Lett. 32, 814 (1974).

[4] N. V. Vitanov, M. Fleischhauer, B. W. Shore, and K. Bergmann, Adv. At., Mol., Opt. Phys. 46, 55 (2001).

[5] P. Marte, P. Zoller, and J. L. Hall, Phys. Rev. A 44, R4118 (1991); M. Weitz, B. C. Young, and S. Chu, Phys. Rev. Lett. 73, 2563 (1994).

[6] R. G. Unanyan, N. V. Vitanov, and K. Bergmann, Phys. Rev. Lett. 87, 137902 (2001).

[7] C. Marr, A. Beige, and G. Rempe, Phys. Rev. A 68, 033817 (2003).
[8] D. Schrader et al., Phys. Rev. Lett. 93, 150501 (2004).

[9] S. Kuhr et al., Science 293, 278 (2001).

[10] D. Schrader et al., Appl. Phys. B: Lasers Opt. 73, 819 (2001).

[11] K. J. Vahala, Nature (London) 424, 839 (2003), and references therein.

[12] I. Dotsenko et al., e-print quant-ph/0411062.

[13] Y. Miroshnychenko et al., Opt. Express 11, 3498 (2003).

[14] S. Guérin, S. Thomas, and H. R. Jauslin, Phys. Rev. A 65, 023409 (2002).

[15] N. V. Vitanov, T. Halfmann, B. W. Shore, and K. Bergmann, Annu. Rev. Phys. Chem. 52, 763 (2001).

[16] S. Kuhr et al., e-print quant-ph/0410037.

[17] L. You, X. X. Yi, and X. H. Su, Phys. Rev. A 67, 032308 (2003). 